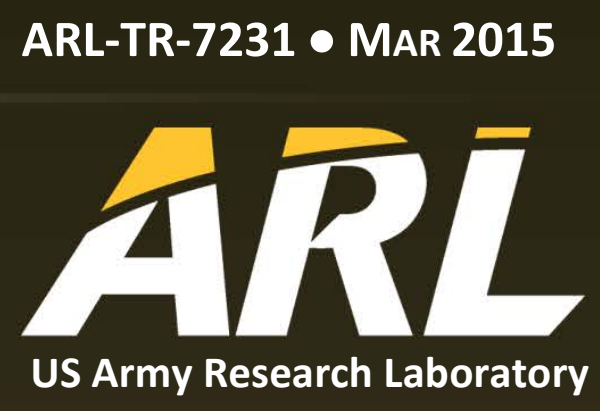

\title{
Reformulation of Nonlinear Anisotropic Crystal Elastoplasticity for Impact Physics
}

by JD Clayton 


\section{NOTICES}

\section{Disclaimers}

The findings in this report are not to be construed as an official Department of the Army position unless so designated by other authorized documents.

Citation of manufacturer's or trade names does not constitute an official endorsement or approval of the use thereof.

Destroy this report when it is no longer needed. Do not return it to the originator. 


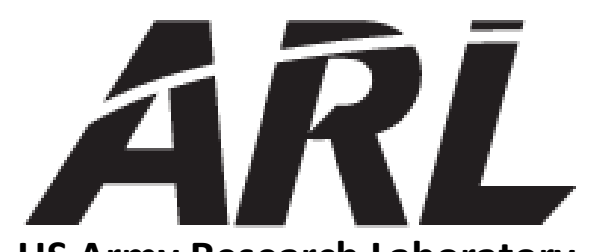

US Army Research Laboratory

\section{Reformulation of Nonlinear Anisotropic Crystal Elastoplasticity for Impact Physics}

\section{by JD Clayton}

Weapons and Materials Research Directorate, ARL 


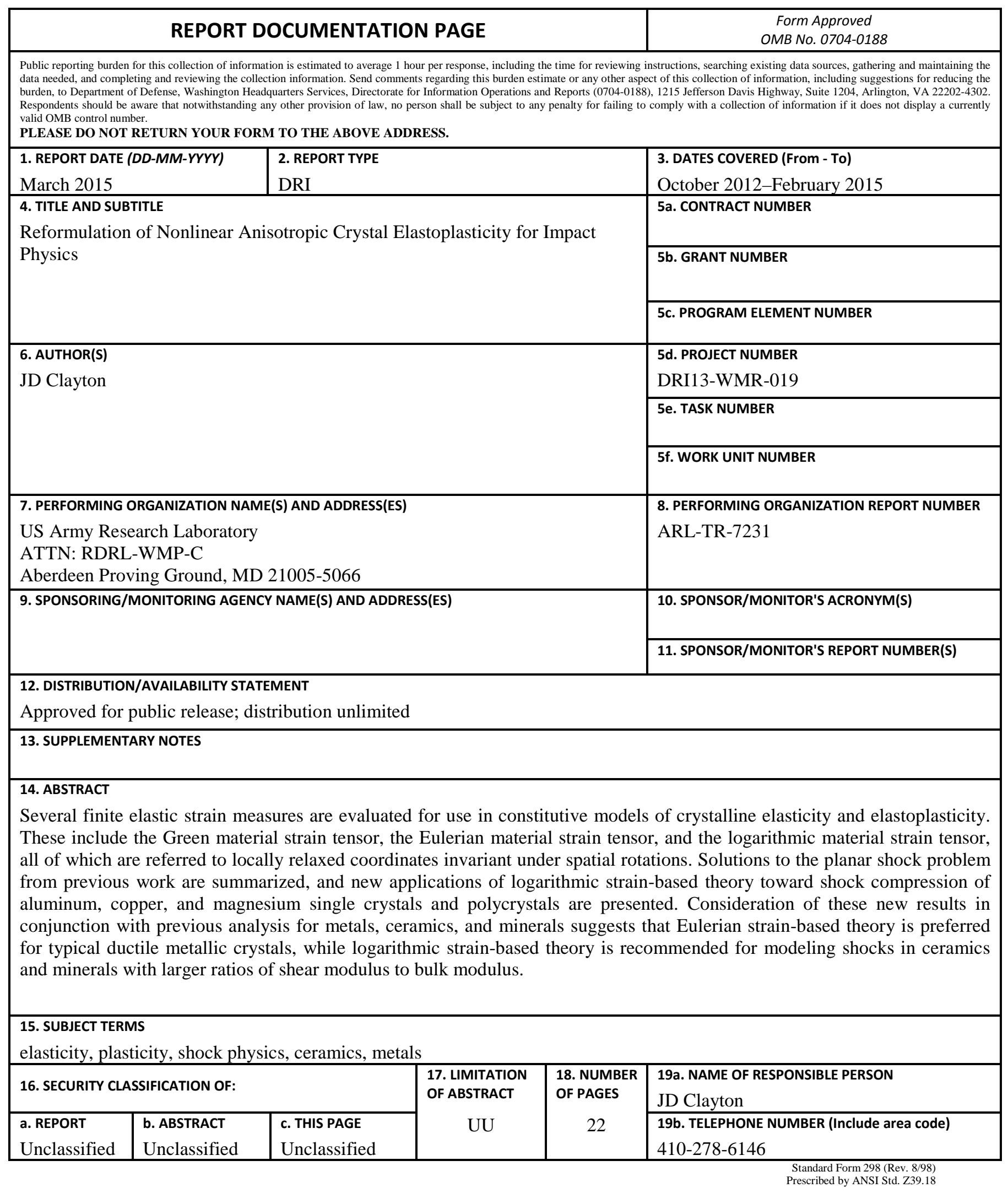




\section{Contents}

List of Figures iv

List of Tables $\quad$ iv

$\begin{array}{ll}\text { 1. Introduction } & 1\end{array}$

2. Approach 3

3. Results 4

4. Conclusions $\quad 8$

5. Transitions 9

$\begin{array}{ll}\text { 6. References } & 10\end{array}$

$\begin{array}{ll}\text { Distribution List } & 15\end{array}$ 


\section{List of Figures}

Fig. 1 Lagrangian $\left(E_{11}\right)$, logarithmic $\left(e_{11}\right)$, and Eulerian $\left(D_{11}\right)$ strains under spherical and uniaxial deformation 2

Fig. 2 Analytical anisotropic thermoelastic solutions for axial stress in (a) shocked Al single crystal compared with atomic simulation data, (b) shocked $\mathrm{Cu}$ single crystal, (c) shocked $\mathrm{Mg}$ single crystal along a-axis, and (d) shocked Mg single crystal along c-axis

Fig. 3 Analytical pressure solutions for polycrystalline (a) aluminum, (b) copper, and (c) magnesium in the hydrodynamic limit compared with

experimental data

\section{List of Tables}

Table 1 Single crystal and polycrystal properties ${ }^{4}\left(\theta_{0}=295 \mathrm{~K} ; \mathrm{C}_{\alpha \beta}\right.$ in GPa; $\rho_{0}$ in $\left.\mathrm{g} / \mathrm{cm}^{3}\right)$

Table 2 Relative error (\%) in shock stress $P$ predicted by third-order (Lag3, Log3, Eul3) and second-order (Lag2, Log2, Eul2) theories: Al and $\mathrm{Cu}$..6

Table 3 Relative error (\%) in shock stress $P$ predicted by third-order (Lag3, Log3, Eul3) and second-order (Lag2, Log2, Eul2) theories: Mg .............6

Table 4 Ratio of ambient Voigt shear modulus to bulk modulus .........................8

Table 5 Summary of present and prior research results: finite strain model evaluations 


\section{Introduction}

Nonlinear hyperelasticity addresses the thermodynamically reversible response of solids subjected to large deformation; classes of crystalline materials of present interest include metals, ceramics, minerals, and energetic materials. Accurate, efficient, stable, and thermodynamically consistent models for nonlinear anisotropic elasticity are required for proper mesoscale modeling of crystalline solids subjected to impact or ballistic loading. At a material element with reference coordinates $\boldsymbol{X}$ and spatial coordinates $\boldsymbol{x}$, the deformation gradient $\boldsymbol{F}$ and volume ratio $J$ are

$$
\boldsymbol{F}(\boldsymbol{X})=\partial \boldsymbol{x} / \partial \boldsymbol{X}, \quad F_{i J}\left(X_{K}\right)=\partial x_{i} / \partial X_{J}=\delta_{i J}+\partial u_{i} / \partial X_{J} ; \quad J(\boldsymbol{X})=V / V_{0}=\operatorname{det} \boldsymbol{F},
$$

where $\boldsymbol{u}$ is the particle displacement vector. For an elastic-plastic material, where "plastic" refers to any thermodynamically irreversible mechanism such as dislocation glide, deformation twinning, fracture, or pore collapse, the total deformation gradient is typically split into a product of a thermoelastic term (superscript $E$ ) and a plastic term (superscript $P$ ): ${ }^{1}$

$$
\boldsymbol{F}=\boldsymbol{F}^{E} \boldsymbol{F}^{P}, \quad F_{i J}=F_{i K}^{E} F_{K J}^{P} ; \quad J=J^{E} J^{P}=\operatorname{det} \boldsymbol{F}^{E} \operatorname{det} \boldsymbol{F}^{P} .
$$

Usual Lagrangian formulations of nonlinear elasticity for crystals ${ }^{1,2}$ incorporate the elastic Green material strain tensor

$$
\boldsymbol{E}=\frac{1}{2}\left(\boldsymbol{F}^{E \mathrm{~T}} \boldsymbol{F}^{E}-\mathbf{1}\right), \quad E_{I J}=\frac{1}{2}\left(F_{k I}^{E} F_{k J}^{E}-\delta_{I J}\right) .
$$

Also considered herein are theories incorporating the elastic Eulerian material strain tensor

$$
\boldsymbol{D}=\frac{1}{2}\left(\mathbf{1}-\boldsymbol{F}^{E-1} \boldsymbol{F}^{E-\mathrm{T}}\right), \quad D_{I J}=\frac{1}{2}\left(\delta_{I J}-F_{l k}^{E-1} F_{J k}^{E-1}\right),
$$

and the elastic material logarithmic strain tensor

$$
\boldsymbol{e}=\ln \boldsymbol{U}^{E}=\frac{1}{2} \ln \left(\boldsymbol{F}^{E \mathrm{~T}} \boldsymbol{F}^{E}\right), \quad \boldsymbol{e}_{I J}=\frac{1}{2} \ln \left(\boldsymbol{F}^{E \mathrm{~T}} \boldsymbol{F}^{E}\right)_{I J} .
$$

Elastic constant tensors of all orders have the same symmetries, though magnitudes of higher-order constants differ among the 3 representations. At fixed entropy, assuming a stress-free reference configuration, and written explicitly with elastic constants up to fourth order, internal energy functions per unit reference volume are expressed as the Taylor polynomials

$$
\bar{U}(\boldsymbol{E})=\frac{1}{2 !} \mathrm{C}_{\alpha \beta} E_{\alpha} E_{\beta}+\frac{1}{3 !} \overline{\mathrm{C}}_{\alpha \beta \gamma} E_{\alpha} E_{\beta} E_{\gamma}+\frac{1}{4 !} \overline{\mathrm{C}}_{\alpha \beta \gamma \delta} E_{\alpha} E_{\beta} E_{\gamma} E_{\delta}+\cdots,
$$




$$
\begin{gathered}
\hat{U}(\boldsymbol{D})=\frac{1}{2 !} \mathrm{C}_{\alpha \beta} D_{\alpha} D_{\beta}+\frac{1}{3 !} \hat{\mathrm{C}}_{\alpha \beta \gamma} D_{\alpha} D_{\beta} D_{\gamma}+\frac{1}{4 !} \hat{\mathrm{C}}_{\alpha \beta \gamma \delta} D_{\alpha} D_{\beta} D_{\gamma} D_{\delta}+\cdots, \\
\breve{U}(\boldsymbol{e})=\frac{1}{2 !} \mathrm{C}_{\alpha \beta} e_{\alpha} e_{\beta}+\frac{1}{3 !} \breve{\mathrm{C}}_{\alpha \beta \gamma} e_{\alpha} e_{\beta} e_{\gamma}+\frac{1}{4 !} \breve{\mathrm{C}}_{\alpha \beta \gamma \delta} e_{\alpha} e_{\beta} e_{\gamma} e_{\delta}+\cdots .
\end{gathered}
$$

Greek indices denote Voigt notation. Consistent relationships among second(equal), third-, and higher-order elastic constants have been derived elsewhere, ${ }^{3-6}$ allowing values for Lagrangian constants entering Eq. 6 to be converted to those in Eqs. 7 and 8 without further experiments. Axial components of strain tensors are compared for spherical and uniaxial deformations in Fig. 1. The magnitude of the axial component of $\boldsymbol{D}$ increases much more rapidly than that of $\boldsymbol{E}$ under compression, with $\boldsymbol{e}$ demonstrating trends intermediate to the other strains. Internal energy, stress/pressure, and stiffness of strong solids all tend to increase rapidly with compression. ${ }^{7}$ Therefore, Eulerian and logarithmic theories would be expected to converge faster, with fewer higher-order elastic constants needed, than Lagrangian theory.

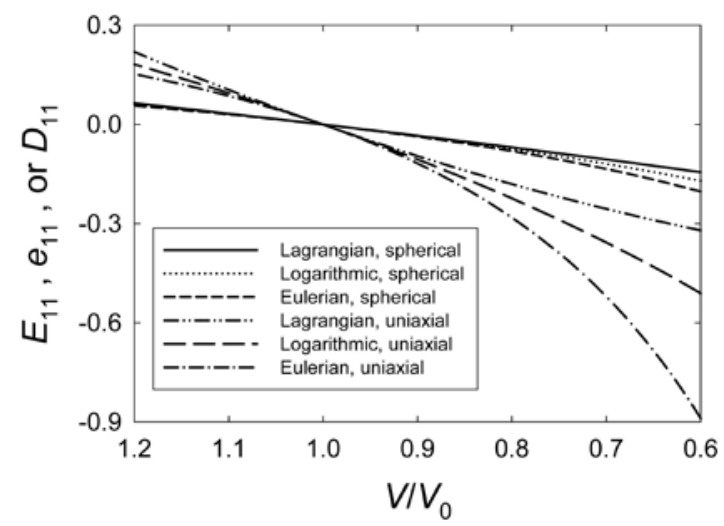

Fig. 1 Lagrangian $\left(E_{11}\right)$, logarithmic $\left(e_{11}\right)$, and Eulerian $\left(D_{11}\right)$ strains under spherical and uniaxial deformation

Benefits of Eulerian strain tensors for isotropic materials were predicted $^{8}$ and demonstrated for cubic crystals under hydrostatic stress. ${ }^{9}$ Thermal effects were considered in Davies ${ }^{10}$ for cubic crystals, and a theory for noncubic crystals was initiated in Weaver. ${ }^{11}$ A complete $\boldsymbol{D}$-based continuum thermoelastic theory for large deformation of crystals of arbitrary symmetry was developed in Clayton. ${ }^{3}$ Analytical solutions for homogeneous deformations of ideal cubic crystals were studied over a prescribed range of elastic coefficients; stress states and intrinsic stability measures were compared. For realistic coefficients, Eulerian theory predicted more realistic and stable behavior than Lagrangian theory under large compression and shear. Analytical solutions for shock compression of anisotropic single crystals were derived for internal energy functions quartic in Lagrangian or Eulerian strain and linear in entropy; results were analyzed for nonmetals quartz, sapphire, and diamond in Clayton ${ }^{3}$ and metals aluminum, copper, and magnesium 
in Clayton. ${ }^{4}$ A complete $\boldsymbol{e}$-based continuum thermoelastic theory was analogously developed in Clayton $^{5}$ and applied to study the shock response of the same 3 nonmetals. The theory was extended to describe elastic-plastic response using the decomposition of Eq. 2, and solutions for plastic shocks (involving slip, twinning, or shear fractures) following an elastic precursor in rate independent solids were derived. ${ }^{5}$ Logarithmic theory delivered superior accuracy to Lagrangian and Eulerian theories for modeling shocks in sapphire (X- and Z-cut), quartz (Z-cut), and diamond (X-cut). ${ }^{5}$ Logarithmic theory incorporating third-order elastic constants was also applied to analytically model the elastic-inelastic response of polycrystalline titanium diboride ceramic, ${ }^{12}$ including double yield and effects of static lateral prestress. Eulerian theory was recently used to numerically model the viscoplastic response of aluminum single crystals and textured polycrystals in wave propagation simulated using the finite difference method, ${ }^{13}$ wherein Lagrangian theory was found insufficient for modeling strong/overdriven elasticplastic shocks.

\section{Approach}

New application of logarithmic strain-based theory (e-based theory) to shock compression of metals is presented here. Predictions are thermoelastic and strictly applicable only for very small volumes, such as in atomic simulations ${ }^{14}$ or in the immediate vicinity of pinned defect cores, ${ }^{15}$ wherein plastic deformation does not occur. Solutions to the planar thermoelastic shock problem in anisotropic crystals were derived fully for Lagrangian and Eulerian theory in Clayton ${ }^{3}$ and for logarithmic theory in Clayton. ${ }^{5}$ The procedure involves simultaneous solution of Rankine-Hugoniot equations for compatibility and conservation of momentum and energy, along with consideration of internal energy functions (Eqs. 6, 7, or 8) extended to include entropy (Grunëisen tensors). Axial shock stress $P$ (positive in compression) is

$$
\begin{aligned}
& P=-J \partial \bar{U} / \partial E=-J\left[\mathrm{C}_{11} E+\frac{1}{2} \overline{\mathrm{C}}_{111} E^{2}+\frac{1}{6} \overline{\mathrm{C}}_{1111} E^{3}+\mathrm{O}\left(E^{3}\right)\right] \quad \text { (Lagrangian), } \\
& P=-J^{-3} \partial \hat{U} / \partial D=-J^{-3}\left[\mathrm{C}_{11} D+\frac{1}{2} \hat{\mathrm{C}}_{111} D^{2}+\frac{1}{6} \hat{\mathrm{C}}_{1111} D^{3}+\mathrm{O}\left(D^{3}\right)\right] \quad \text { (Eulerian), } \\
& P=-J^{-1} \partial \breve{U} / \partial e=-J^{-1}\left[\mathrm{C}_{11} e+\frac{1}{2} \breve{\mathrm{C}}_{111} e^{2}+\frac{1}{6} \breve{\mathrm{C}}_{1111} e^{3}+\mathrm{O}\left(e^{3}\right)\right] \quad \text { (Logarithmic), }
\end{aligned}
$$

where entropic contributions are of order 3 in scalar strain measures for uniaxial strain:

$$
J=(1+2 E)^{1 / 2}=(1-2 D)^{-1 / 2}=\exp (e) .
$$


In laboratory-scale specimens, yielding would commence in pure ductile metals at small compressions at which effects of higher-order constants and differences among Eqs. 9-11 would be negligible. However, nonlinear elastic effects on deviatoric stress may be important at larger compressions after yielding, particularly for lower symmetry materials with restricted slip planes/directions, ${ }^{4}$ and the nonlinearity in pressure-volume response is important regardless of shear strength. When strength is low and can safely be omitted, the response of metals to moderate shocks can often be adequately approximated $(p \approx P)$ by an adiabatic pressure-volume $(p-J)$ equation of state (EOS) for spherical compression. The EOS corresponding to each of Eqs. 6-8 truncated at order 3 in strain is ${ }^{3-5}$

$$
\begin{aligned}
& p=-\partial \bar{U} / \partial J=\frac{3}{2} \mathrm{~B}_{0}\left(J^{-1 / 3}-J^{1 / 3}\right)\left[1-\frac{3}{4} \mathrm{~B}_{0}^{\prime}\left(J^{2 / 3}-1\right)\right] \\
& p=-\partial \hat{U} / \partial J=\frac{3}{2} \mathrm{~B}_{0}\left(J^{-7 / 3}-J^{-5 / 3}\right)\left[1+\frac{3}{4}\left(\mathrm{~B}_{0}^{\prime}-4\right)\left(J^{-2 / 3}-1\right)\right] \\
& p=-\partial \breve{U} / \partial J=-\mathrm{B}_{0}[(\ln J) / J]\left[1-\frac{1}{2}\left(\mathrm{~B}_{0}^{\prime}-2\right) \ln J\right]
\end{aligned}
$$

where $B_{0}$ is the isentropic bulk modulus and $B_{0}^{\prime}$ is its pressure derivative in the reference state.

\section{Results}

Properties for aluminum $(\mathrm{Al})$, copper $(\mathrm{Cu})$, and magnesium $(\mathrm{Mg})$ are reported in Table 1. ${ }^{4}$ Predictions for shock stress versus volume ratio are made using the analytical solutions of Eqs. 9-11, considering only pure mode directions (strictly longitudinal response). Shock stress $P$ normalized by second-order isentropic elastic constant $\mathrm{C}_{11}$ is shown in Fig. 2a for $\mathrm{Al}$, Fig. 2b for $\mathrm{Cu}$, Fig. 2c for $\mathrm{Mg}$ [a-axis] and Fig. 2d for Mg [c-axis]. Higher-order elastic constants are converted from measured/predicted Lagrangian constants using formulae derived or presented in Clayton $^{3-5}$ and Perrin and Delannoy-Coutris. ${ }^{6}$ Elastic constants of up to order 4 are considered in results labeled "4th order". Results labeled "3rd order" and "2nd order" are obtained, respectively, by setting fourth-order and both third- and fourth-order elastic constants to zero. Results in Fig. 2 compare logarithmic and Eulerian theories; plots comparing Lagrangian and Eulerian theories can be found in Clayton. ${ }^{4}$ All longitudinal higher-order elastic constants (i.e., all third- and fourth-order constants) are smaller in magnitude for Eulerian and logarithmic theory than Lagrangian theory for these metals (Table 1). Stress predictions of second- and third-order models are usually closer to those of fourth-order theory for Eulerian and logarithmic theory than Lagrangian theory. Tables 2 and 3 list relative error (\%) of second- and third-order predictions 
relative to fourth-order predictions, computed as $2 \times$ (second- or third-order result - fourth-order result)/(second- or third-order result + fourth-order result). For each crystal type, such errors are almost always smaller in magnitude for Eulerian theory (Eul2, Eul3) and logarithmic theory (Log2, Log3) than for Lagrangian theory (Lag2, Lag3) at a given volume ratio and order of approximation. Together, these observations imply a faster converging series in Eqs. 7 or 8 than in Eq. 6, as the number of higher-order constants is increased and greater accuracy of Eulerian or logarithmic theory than Lagrangian when the same number of constants (i.e., the same order of Taylor polynomial) is used. Eulerian and logarithmic theories trend toward comparable accuracy with one theory or the other possibly more accurate for a given metal or order of approximation. For $\mathrm{Al}$, as shown in Fig. 2a, the analytical solution incorporating fourth-order Eulerian theory best matches atomic predictions: ${ }^{14}$ at $J=0.923$, error in fourth-order Eulerian theory versus atomic simulation is $-1.4 \%$, compared to $-7.4 \%$ for fourthorder Lagrangian theory and $-2.2 \%$ for fourth-order logarithmic theory.

Table 1 Single crystal and polycrystal properties ${ }^{4}\left(\theta_{0}=295 \mathrm{~K}\right.$; $\mathrm{C}_{\alpha \beta}$ in GPa; $\rho_{0}$ in $\left.\mathrm{g} / \mathrm{cm}^{3}\right)$

\begin{tabular}{lcccc}
\hline Property & Al [100] & Cu [100] & Mg [a-axis] Mg [c-axis] \\
\hline $\mathrm{C}_{11}$ & 107 & 166 & 59.4 & 61.6 \\
$\overline{\mathrm{C}}_{111}$ & $-1,080$ & $-1,279$ & -664 & -728 \\
$\hat{\mathrm{C}}_{111}$ & 203 & 715 & 49 & 12 \\
$\breve{\mathrm{C}}_{111}$ & -438 & -283 & -308 & -358 \\
$\overline{\mathrm{C}}_{1111}$ & 25,000 & 11,900 & 8,170 & 7,380 \\
$\hat{\mathrm{C}}_{1111}$ & 10,500 & 2,000 & 1,220 & 893 \\
$\breve{\mathrm{C}}_{1111}$ & 15,036 & 1,200 & 1,865 & 369 \\
$\Gamma_{1}$ & 2.17 & 1.97 & 1.52 & 1.52 \\
$\mathrm{~B}_{0}$ & 76 & 137 & 35.4 & 35.4 \\
$\mathrm{~B}_{0}^{\prime}$ & 4.42 & 5.48 & 3.90 & 3.90 \\
$\rho_{0}$ & 2.70 & 8.96 & 1.74 & 1.74 \\
\hline
\end{tabular}



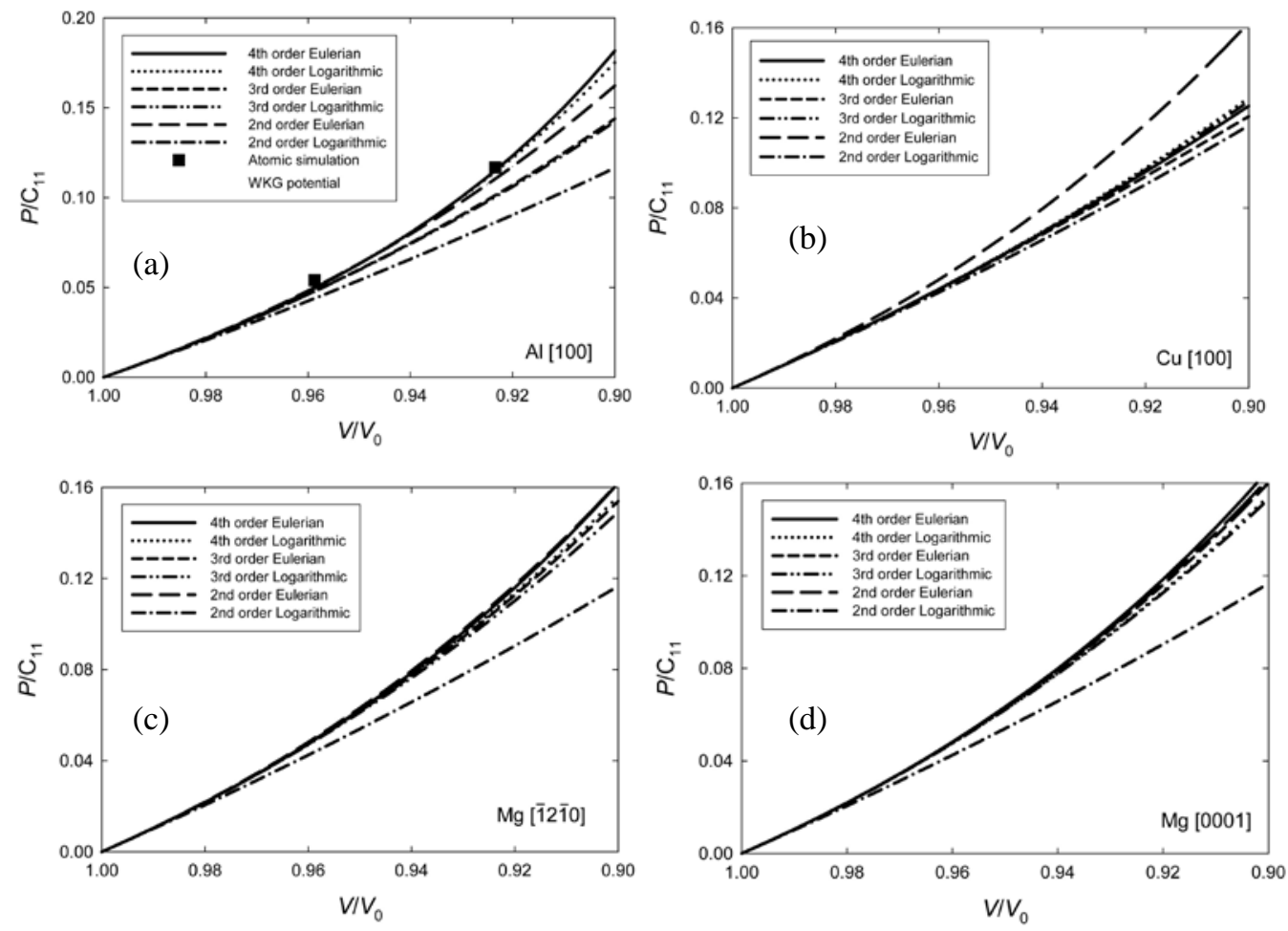

Fig. 2 Analytical anisotropic thermoelastic solutions for axial stress in (a) shocked Al single crystal compared with atomic simulation data, ${ }^{16}$ (b) shocked $\mathrm{Cu}$ single crystal, (c) shocked Mg single crystal along a-axis, and (d) shocked Mg single crystal along c-axis

Table 2 Relative error (\%) in shock stress $P$ predicted by third-order (Lag3, Log3, Eul3) and second-order (Lag2, Log2, Eul2) theories: $\mathrm{Al}$ and $\mathrm{Cu}$

\begin{tabular}{c|cccccc|cccccc}
\hline \multirow{2}{*}{$\boldsymbol{V} / \boldsymbol{V}_{\mathbf{0}}$} & \multicolumn{5}{|c|}{ Aluminum [100] } & \multicolumn{5}{c}{ Copper [100] } \\
\cline { 2 - 14 } & Lag3 & Log3 & Eul3 & Lag2 & Log2 & Eul2 & Lag3 & Log3 & Eul3 & Lag2 & Log2 & Eul2 \\
\hline 0.96 & -5.0 & -3.8 & -3.1 & -23.1 & -11.9 & +1.1 & -1.6 & -0.2 & -0.4 & -15.8 & -3.7 & +9.3 \\
0.92 & -15.7 & -13.9 & -14.1 & -48.2 & -29.8 & -5.0 & -5.4 & -0.8 & -2.1 & -31.6 & -7.8 & +19.9 \\
0.88 & -28.1 & -28.2 & -34.5 & -71.2 & -51.0 & -19.8 & -10.4 & -1.8 & -6.2 & -46.5 & -12.4 & +31.5 \\
\hline
\end{tabular}

Table 3 Relative error (\%) in shock stress $P$ predicted by third-order (Lag3, Log3, Eul3) and second-order (Lag2, Log2, Eul2) theories: Mg

\begin{tabular}{c|cccccc|cccccc}
\hline \multirow{2}{*}{$\boldsymbol{V} / \mathbf{V}_{\mathbf{0}}$} & \multicolumn{5}{|c|}{ Magnesium [a-axis] } & \multicolumn{5}{c}{ Magnesium [c-axis] } \\
\cline { 2 - 11 } & Lag3 & Log3 & Eul3 & Lag2 & Log2 & Eul2 & Lag3 & Log3 & Eul3 & Lag2 & Log2 & Eul2 \\
\hline 0.96 & -2.9 & -0.8 & -0.6 & -22.8 & -10.9 & 1.2 & -2.5 & -0.2 & -0.4 & -23.4 & -11.0 & -0.0 \\
0.92 & -9.2 & -3.0 & -2.9 & -44.7 & -22.7 & 1.0 & -7.9 & -0.6 & -2.0 & -45.1 & -22.5 & -1.2 \\
0.88 & -16.8 & -6.3 & -7.6 & -64.3 & -35.0 & -1.4 & -14.5 & -1.2 & -5.2 & -64.2 & -33.0 & -3.8 \\
\hline
\end{tabular}


Predictions of each third-order EOS in Eqs. 13-15 are compared with shock compression data ${ }^{16}$ for $\mathrm{Al}$ in Fig. 3a, $\mathrm{Cu}$ in Fig. 3b, and $\mathrm{Mg}$ in Fig. 3c. Compressibility properties of Table 1 used in these predictions are obtained from ultrasonic experiments ${ }^{17}$ and are not fit to the shock data. In each case, Eulerian theory is most accurate, followed by logarithmic theory and then Lagrangian theory, the latter significantly too compliant. Because Eulerian theory appears superior for modeling spherical compression and at least equally valid as logarithmic theory for modeling uniaxial compression, Eulerian theory is preferred overall for representing the shock response of metals. Future work should consider incorporating Eulerian theory in finite element simulations of the high-rate response of metals, offering potential improvement over prior implementations involving Lagrangian theory. ${ }^{18-20}$ Eulerian EOS (Eq. 14) is equivalent to the Birch-Murnaghan EOS. ${ }^{9}$ However, recent work $^{5}$ found that logarithmic theory, while more computationally cumbersome than Eulerian theory, more accurately captures the shock response of ceramic crystals sapphire $\left(\alpha-\mathrm{Al}_{2} \mathrm{O}_{3}\right)$, diamond $(\mathrm{C})$, and quartz $\left(\alpha-\mathrm{SiO}_{2}\right)$. As shown in Table 4 , these nonmetals have a large ratio of effective shear $\left(\mathrm{G}_{0}\right)$ to bulk modulus compared to metals.
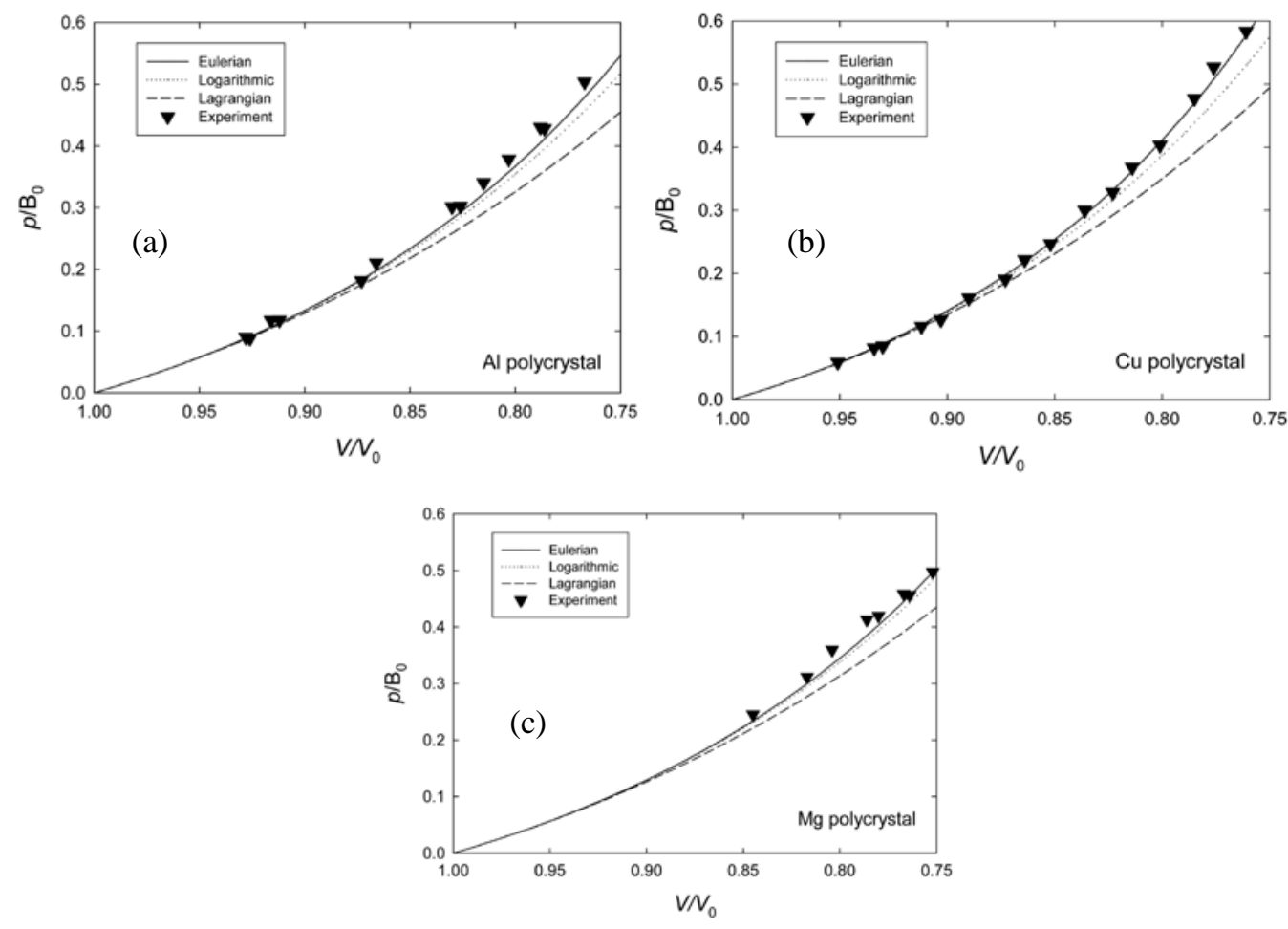

Fig. 3 Analytical pressure solutions for polycrystalline (a) aluminum, (b) copper, and (c) magnesium in the hydrodynamic limit compared with experimental data ${ }^{16}$ 
Table 4 Ratio of ambient Voigt shear modulus to bulk modulus

\begin{tabular}{ccccccc}
\hline Ratio & $\mathbf{C u}$ & $\mathbf{A l}$ & $\mathbf{M g}$ & $\boldsymbol{\alpha}-\mathbf{A l}_{2} \mathbf{O}_{3}$ & $\mathbf{C}$ & $\boldsymbol{\alpha}-\mathbf{S i O}_{2}$ \\
\hline $\mathrm{G}_{0} / \mathrm{B}_{0}$ & 0.34 & 0.35 & 0.53 & 0.65 & 1.22 & 1.27 \\
\hline
\end{tabular}

\section{Conclusions}

Table 5 summarizes loading conditions, materials, and model performance studied in the present work as well as in Clayton. ${ }^{3-5}$ Eulerian nonlinear theory developed in Clayton ${ }^{3,4}$ is recommended for modeling the response of ductile metals, while logarithmic theory developed in Clayton ${ }^{5}$ can be recommended for modeling ceramics and minerals with larger ratios of shear to bulk stiffness.

Table 5 Summary of present and prior research results: finite strain model evaluations

\begin{tabular}{|c|c|c|c|c|}
\hline Loading Protocol & Material & $\begin{array}{l}\text { Recommended } \\
\text { Theory }\end{array}$ & Remarks & Ref. \\
\hline $\begin{array}{l}\text { Hydrostatic } \\
\text { compression }\end{array}$ & $\begin{array}{l}\text { Ideal cubic, } \\
\mathrm{B}_{0}^{\prime}=4\end{array}$ & Eulerian & $\begin{array}{l}\text { More accurate } p-V \\
\text { response }\end{array}$ & 3 \\
\hline $\begin{array}{l}\text { Uniaxial } \\
\text { compression }\end{array}$ & $\begin{array}{l}\text { Ideal cubic, } \\
\mathrm{B}^{\prime}{ }_{0}=4\end{array}$ & Eulerian & More accurate and stable & 3 \\
\hline Simple shear & $\begin{array}{l}\text { Ideal cubic, } \\
\mathrm{B}_{0}^{\prime}=4\end{array}$ & Eulerian & More accurate and stable & 3 \\
\hline \multirow{6}{*}{ Shock compression } & Sapphire & \multirow{3}{*}{ Logarithmic } & More accurate overall & 5 \\
\hline & Diamond & & More accurate overall & 5 \\
\hline & $\alpha$-Quartz & & More accurate for Z-cut & 3,5 \\
\hline & Aluminum & \multirow{3}{*}{ Eulerian } & Best fit to atomic data & $4^{\mathrm{a}}$ \\
\hline & Copper & & Faster convergence & $4^{\mathrm{a}}$ \\
\hline & Magnesium & & Faster convergence & $4^{\mathrm{a}}$ \\
\hline
\end{tabular}

${ }^{\mathrm{a}}$ Present work 


\section{Transitions}

Results of the current work are of high interest to modeling communities within the Department of Defense, Department of Energy, and the Materials in Extreme Dynamic Environments Collaborative Research Alliance. Results have been transitioned via publications. ${ }^{3-5,12,13,15}$ A plan is underway to implement the model into multiscale simulations of armor and munitions at the US Army Research Laboratory. Specifically, developments from this Director's Research Initiative (DRI) project are expected to offer substantial improvements over prior analytical and computational studies of the finite strain response of metals, ${ }^{18-26}$ ceramics, $^{27-}$ 33 concrete and geologic materials, ${ }^{34,35}$ and energetic molecular crystals. ${ }^{36}$ The nonlinear elastic theory can be directly implemented into phase-field simulations of microstructure. ${ }^{37-41} \mathrm{New}$ developments in studies of lattice defects in electronic materials $^{42-44}$ and generic crystalline solids ${ }^{45-55}$ are also foreseen. Results of Year 1 of this DRI project were reported in Clayton. ${ }^{56}$ 


\section{References}

1. Clayton JD. Nonlinear mechanics of crystals. Dordrecht (The Netherlands): Springer; 2011.

2. Wallace DC. Statistical physics of crystals and liquids: a guide to highly accurate equations of state. Singapore: World Scientific; 2003.

3. Clayton JD. Nonlinear Eulerian thermoelasticity for anisotropic crystals. Journal of the Mechanics and Physics of Solids. 2013;61:1983-2014.

4. Clayton JD. Shock compression of metal crystals: a comparison of Eulerian and Lagrangian elastic-plastic theories. International Journal of Applied Mechanics. 2014;6:1450048.

5. Clayton JD. Analysis of shock compression of strong single crystals with logarithmic thermoelastic-plastic theory. International Journal of Engineering Science. 2014;79:1-20.

6. Perrin G, Delannoy-Coutris M. Analysis of plane elastic-plastic shock-waves from the fourth-order anharmonic theory. Mechanics of Materials. 1983;2:139-153.

7. Jeanloz R. Shock wave equation of state and finite strain theory. Journal of Geophysical Research. 1989;94:5873-5886.

8. Murnaghan FD. Finite deformations of an elastic solid. American Journal of Mathematics. 1937;59:235-260.

9. Birch F. Finite elastic strain of cubic crystals. Physical Review. 1947;71:809-824.

10. Davies GF. Effective elastic moduli under hydrostatic stress-I quasiharmonic theory. Journal of the Physics and Chemistry of Solids. 1974;35:1513-1520.

11. Weaver JS. Application of finite strain theory to non-cubic crystals. Journal of the Physics and Chemistry of Solids. 1976;37:711-718.

12. Clayton JD. Finite strain analysis of shock compression of brittle solids applied to titanium diboride. International Journal of Impact Engineering. 2014;73:56-65. 
13. Lloyd JT, Clayton JD, Becker R, and McDowell DL. Simulation of shock wave propagation in single crystal and polycrystalline aluminum. International Journal of Plasticity. 2014;60:118-144.

14. Zimmerman JA, Winey JM, Gupta YM. Elastic anisotropy of shocked aluminum single crystals: use of molecular dynamics simulations. Physical Review B. 2011;83:184113.

15. Clayton JD. Defects in nonlinear elastic crystals: differential geometry, finite kinematics, and second-order analytical solutions. Zeitschrift für Angewandte Mathematik und Mechanik (ZAMM). 2013 Oct 28. doi: 10.1002 /zamm.201300142.

16. Marsh SP. LASL Shock Hugoniot data. Berkeley (CA): Univ. of California Press; 1980.

17. Guinan MW, Steinberg DJ. Pressure and temperature derivatives of the isotropic polycrystalline shear modulus for 65 elements. Journal of the Physics and Chemistry of Solids. 1974;35:1501-1512.

18. Clayton JD, McDowell DL. A multiscale multiplicative decomposition for elastoplasticity of polycrystals. International Journal of Plasticity. 2003;19:1401-1444.

19. Clayton JD. Dynamic plasticity and fracture in high density polycrystals: constitutive modeling and numerical simulation. Journal of the Mechanics and Physics of Solids. 2005;53:261-301.

20. Clayton JD. Modeling dynamic plasticity and spall fracture in high density polycrystalline alloys. International Journal of Solids and Structures. 2005;42:4613-4640.

21. Clayton JD, Schroeter BM, Graham S, McDowell DL. Distributions of stretch and rotation in OFHC Cu. Journal of Engineering Materials and Technology. 2002;124:302-313.

22. Clayton JD, McDowell DL. Homogenized finite elastoplasticity and damage: theory and computations. Mechanics of Materials. 2004;36:799-824.

23. Clayton JD. Continuum multiscale modeling of finite deformation plasticity and anisotropic damage in polycrystals. Theoretical and Applied Fracture Mechanics. 2006;45:163-185. 
24. Vogler TJ, Clayton JD. Heterogeneous deformation and spall of an extruded tungsten alloy: plate impact experiments and crystal plasticity modeling. Journal of the Mechanics and Physics of Solids. 2008;56:297-335.

25. Clayton JD. Modeling effects of crystalline microstructure, energy storage mechanisms, and residual volume changes on penetration resistance of precipitate-hardened aluminum alloys. Composites B: Engineering. 2009;40:443-450.

26. Clayton JD. Two-scale modeling of effects of microstructure and thermomechanical properties on the dynamic performance of an aluminum alloy. International Journal of Materials and Structural Integrity. 2010;4:116140 .

27. Clayton JD. A continuum description of nonlinear elasticity, slip and twinning, with application to sapphire. Proceedings of the Royal Society A, 2009;465:307-334.

28. Clayton JD. Modeling finite deformations in trigonal ceramic crystals with lattice defects. International Journal of Plasticity. 2010;26:1357-1386.

29. Clayton JD. Modeling nonlinear electromechanical behavior of shocked silicon carbide. Journal of Applied Physics. 2010;107:013520.

30. Clayton JD. A nonlinear thermomechanical model of spinel ceramics applied to aluminum oxynitride (AlON). Journal of Applied Mechanics. 2011;78:011013.

31. Clayton JD. Towards a nonlinear elastic representation of finite compression and instability of boron carbide ceramic. Philosophical Magazine. 2012;92:2860-2893.

32. Clayton JD, Kraft RH, Leavy RB. Mesoscale modeling of nonlinear elasticity and fracture in ceramic polycrystals under dynamic shear and compression. International Journal of Solids and Structures. 2012;49:2686-2702.

33. Clayton JD. Mesoscale modeling of dynamic compression of boron carbide polycrystals. Mechanics Research Communications. 2013;49:57-64.

34. Clayton JD. A model for deformation and fragmentation in crushable brittle solids. International Journal of Impact Engineering. 2008;35:269-289.

35 Clayton JD. Deformation, fracture, and fragmentation in brittle geologic solids. International Journal of Fracture. 2010;163:151-172. 
36. Clayton JD, Becker R. Elastic-plastic behavior of cyclotrimethylene trinitramine single crystals under spherical indentation: modeling and simulation. Journal of Applied Physics. 2012;111:063512.

37. Clayton JD, Knap J. A phase field model of deformation twinning: nonlinear theory and numerical simulations. Physica D. 2011;240:841-858.

38. Clayton JD, Knap J. Phase field modeling of twinning in indentation of transparent single crystals. Modelling and Simulation in Materials Science and Engineering. 2011;19:085005.

39. Clayton JD, Knap J. Phase field analysis of fracture induced twinning in single crystals. Acta Materialia. 2013;61:5341-5353.

40. Clayton JD. Phase field theory and analysis of pressure-shear induced amorphization and failure in boron carbide ceramic. AIMS Materials Science. 2014;1:143-158.

41. Clayton JD, Knap J. Phase field modeling of directional fracture in anisotropic polycrystals. Computational Materials Science. 2015;98:158169.

42. Clayton JD, Chung PW, Grinfeld MA, Nothwang WD. Continuum modeling of charged vacancy migration in elastic dielectric solids, with application to perovskite thin films. Mechanics Research Communications. 2008;35:57-64.

43. Clayton JD, Chung PW, Grinfeld MA, Nothwang WD. Kinematics, electromechanics, and kinetics of dielectric and piezoelectric crystals with lattice defects. International Journal of Engineering Science. 2008;46:10-30.

44. Clayton JD. A non-linear model for elastic dielectric crystals with mobile vacancies. International Journal of Non-linear Mechanics. 2009;44:675-688.

45. Clayton JD, McDowell DL, Bammann DJ. A multiscale gradient theory for elastoviscoplasticity of single crystals. International Journal of Engineering Science. 2004;42:427-457.

46. Clayton JD, Bammann DJ, McDowell DL. Anholonomic configuration spaces and metric tensors in finite strain elastoplasticity. International Journal of Non-Linear Mechanics. 2004;39:1039-1049.

47. Clayton JD, Bammann DJ, McDowell DL. A geometric framework for the kinematics of crystals with defects. Philosophical Magazine. 2005;85:39834010 . 
48. Clayton JD, McDowell DL, Bammann DJ. Modeling dislocations and disclinations with finite micropolar elastoplasticity. International Journal of Plasticity. 2006;22:210-256.

49. Clayton JD, Chung PW. An atomistic-to-continuum framework for nonlinear crystal mechanics based on asymptotic homogenization. Journal of the Mechanics and Physics of Solids. 2006;54:1604-1639.

50. Chung PW, Clayton JD. Multiscale modeling of point and line defects in cubic crystals. International Journal of Multiscale Computational Engineering. 2007;5:203-226.

51. Clayton JD, Bammann DJ. Finite deformations and internal forces in elasticplastic crystals: interpretations from nonlinear elasticity and anharmonic lattice statics. Journal of Engineering Materials and Technology. 2009;131:041201.

52. Clayton JD. On anholonomic deformation, geometry, and differentiation. Mathematics and Mechanics of Solids. 2012;17:702-735.

53. Clayton JD, Hartley CS, McDowell DL. The missing term in the decomposition of finite deformation. International Journal of Plasticity. 2014;52:51-76.

54. Clayton JD. An alternative three-term decomposition for single crystal deformation motivated by non-linear elastic dislocation solutions. Quarterly Journal of Mechanics and Applied Mathematics. 2014;67:127-158.

55. Clayton JD. Differential geometry and kinematics of continua. Singapore: World Scientific; 2014.

56. Clayton JD. A reformulation of nonlinear anisotropic elasticity for impact physics. Aberdeen Proving Ground (MD): Army Research Laboratory (US); 2014. Report No.: ARL-TR-6837. Also available at http://www.arl.army.mil/www/default.cfm?technical_report=7009. 


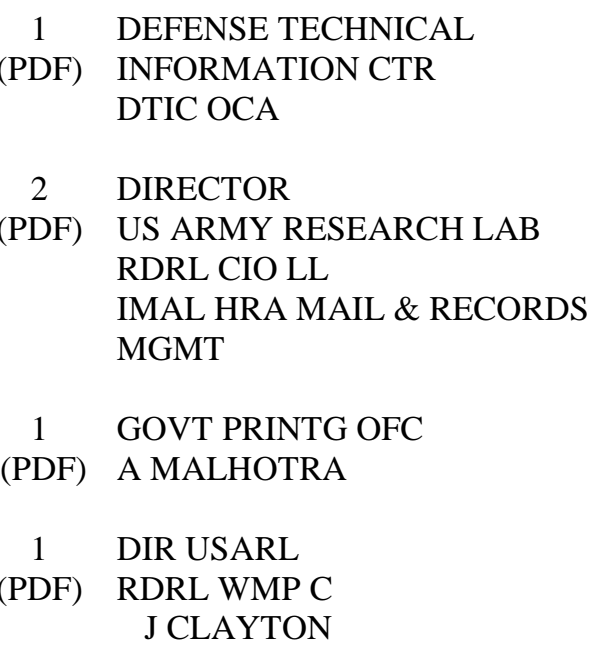


INTENTIONALLY LEFT BLANK. 